Research Paper

\title{
In Vivo Cancer Targeting and Imaging-Guided Surgery with Near Infrared-Emitting Quantum Dot Bioconjugates
}

\author{
Yan $\mathrm{Li}^{1^{*}}$, Zhe Li ${ }^{2}$, Xiaohui Wang ${ }^{3}$, Fengjun Liu ${ }^{3}$, Yingsheng Cheng ${ }^{3}$, Bingbo Zhang ${ }^{4}{ }^{凶}$, and Donglu Shi 4,5 \\ 1. Translational Medicine Research Centre \& Central Laboratory, Shanghai First Maternity and Infant Hospital, Tongji \\ University School of Medicine, Shanghai, 200040, P.R. China. \\ 2. Department of Thyroid and Breast Surgery, the Tenth People' s Hospital of Shanghai, Tongji University, Shanghai 200072, \\ P. R. China \\ 3. Medical Imaging Center, the Tenth People's Hospital of Shanghai, Tongji University, Shanghai 200072, P. R. China. \\ 4. The Institute for Advanced Materials and Nano Biomedicine, Tongji University, Shanghai, 200092, P. R. China. \\ 5. School of Electronic and Computing Systems, University of Cincinnati, Cincinnati, Ohio 45221, USA.
}

* These two authors contribute equally to this study.

$\triangle$ Corresponding author: Bingbo Zhang: bingbozhang@tongji.edu.cn. Tel: (+) 86-21-65983706-819. Fax: (+) 86-21-65983706-0.

(c) Ivyspring International Publisher. This is an open-access article distributed under the terms of the Creative Commons License (http://creativecommons.org/ licenses/by-nc-nd/3.0/). Reproduction is permitted for personal, noncommercial use, provided that the article is in whole, unmodified, and properly cited.

Received: 2012.06.04; Accepted: 2012.07.02; Published: 2012.08.07

\begin{abstract}
Early detection and subsequent complete surgical resection are among the most efficient methods for treating cancer. However, low detection sensitivity and incomplete tumor resection are two challenging issues. Nanoparticle-based imaging-guided surgery has proven promising for cancer-targeted imaging and subsequent debulking surgery. Particularly, the use of near infrared (NIR) fluorescent probes such as NIR quantum dots (QDs) allows deep penetration and high sensitivity for tumor detection. In this study, NIR-emitting CdTe QDs (maximum fluorescence emission peak at $728 \mathrm{~nm}$ ) were synthesized with a high quantum yield (QY) of 38\%. The tumor-specific QD bioconjugates were obtained by attaching cyclic Arg-Gly-Asp peptide (cRGD) to the surface of synthesized QDs, and then injected into U87 MG tumor-bearing mice via tail veins for tumor-targeted imaging. The tumor and its margins were visualized and distinguished by NIR QD bioconjugates, and tumor resection was successfully accomplished via NIR guidance using a Fluobeam-700 NIR imaging system. Our work indicates that the synthesized tumor-specific NIR QDs hold great promise as a potential fluorescent indicator for intraoperative tumor imaging.
\end{abstract}

Key words: Imaging-guided surgery; NIR quantum dots; cancer treatment; cancer-targeted imaging; RGD

\section{Introduction}

Surgical therapy is one of the most frequently used solutions for cancer treatment. In cancer surgery, intraoperative assessment of surgical margins mainly relies on the visual appearance and palpation of tumor subjectively acquired by the surgeons. Consequently, the surgeons inevitably encounter difficulties in tumor resection regarding determination of the tumor surgical margins surrounding severely-mutilated normal tissues and subsequent complete tumor resection $[1,2]$. It has been reported that the rate of tumor recurrence can reach $20-30 \%$ after its removal by surgical procedures, and subsequent cancer metastasis can further lead to clinical complications $[3,4]$. 
In order to improve the surgical quality and the prognosis of patients, the surgeons need to maximize tumor removal, minimize peritumoral damage to the surrounding normal tissues and shorten surgical time. The optical imaging techniques provide real-time tumor visualization, thus allowing intraoperative image-guided surgery (IGS) $[2,5,6]$. Currently, indocyanine green (ICG) is an imaging contrast agent commonly used for optical IGS in hospitals. However, the application of ICG has been limited largely due to several of its physicochemical characteristics, such as the concentration-dependent aggregation, the poor aqueous stability, the nonspecific binding to proteins, and the lack of targeting specificity [7]. These can lead to a quick clearance of ICG by liver with a short half-life of several minutes.

Nanomaterials and nanotechnology for diagnosis and/or treatment of cancers have received an increasing attention from scientists in materials, biology, chemistry and medicine [8-10]. Associated techniques have been shown effective and promising for treating cancers [10-12]. Dedicated nanomaterials have been employed for in vivo detection of tumor biomarkers in blood, cancer cells and tiny solid tumors [13-15]. Nanotechnology-based optical IGS is becoming a promising trend in cancer treatment [16]. Recently, there has been a surge in the development of nanoparticles doped with organic dyes for imaging. Such nanoencapsulation can improve the stability of organic dyes, further amplifying their signal intensity. In addition, post-surface modification of nanoparticles can improve their hydrophily and resistance to nonspecific binding. However, organic dyes have one inherent disadvantage regarding fluorescence quenching and bleaching, which is a fatal blow during intraoperative imaging.

Quantum dots (QDs) are novel fluorophores comprised of elements from groups II-VI, III-V or I-III-VI in the periodic table. As compared with traditional organic dyes, QDs are advantageous due to their wavelength-tunable emission and broad excitation spectra, sharp and symmetrical fluorescent peak, large Stokes shift and high photostability [17-20]. The QDs have increasingly been used in promising biological applications, especially as fluorescent probes for tumor imaging and detection [21-23]. Due to the inherent photostability, QDs have high resistance to photobleaching and allow long-time visualization for in vivo intraoperative imaging guidance [24].

NIR fluorescence imaging is the latest trend in IGS applications, as it is convenient, non-invasive and real-time image-guided during the surgery, without changing the appearance of the surgical field [16]. As compared with visible fluorophores, NIR fluoro- phores have more advantages due to the negligible absorption and autofluorescence of living tissues in the NIR wavelength region [25]. To overcome the incomplete tumor resection and disadvantages of current fluorophores, this study developed a novel class of NIR nanoparticles for application of IGS. A tumor targeting peptide was selected and attached to the surface of NIR nanoparticles, and tumor-bearing mice were used as the animal model to show the preferential accumulation of cRGD-functionalized NIR nanoparticles at the tumor site. The advantages of NIR nanoparticle-guided resection versus simple visual resection of tumor in mice were demonstrated and discussed.

\section{Materials and methods}

\section{I Chemicals}

Sodium borohydride $\left(\mathrm{NaBH}_{4}, 99 \%\right)$, tellurium powder (200 mesh, 99.8\%), cadmium chloride hemi(pentahydrate $) \quad\left(\mathrm{CdCl}_{2},>99 \%\right), \quad$ and 3-mercaptopropionic acid (MPA, 99\%) were purchased from Sigma-Aldrich (US), cyclic Arg-Gly-Asp (cRGD) peptide purchased from Apeptide (Shanghai, China), and 1-Ethyl-3-(3-dimethyllaminopropyl) carbodiimide hydrochloride $(\mathrm{EDC} \cdot \mathrm{HCl})$ purchased from GL Biochem (Shanghai, China). Deionized water (18.2 $\mathrm{M} \Omega \bullet \mathrm{cm}$ resistivity at $25^{\circ} \mathrm{C}$ ) was used for all experiments.

\subsection{Synthesis of NIR CdTe QDs}

The Te source (NaHTe) was prepared following a modified protocol [26]. First, $50.8 \mathrm{mg}$ of tellurium powder and $37.8 \mathrm{mg}$ of $\mathrm{NaBH}_{4}$ were loaded into a 25 $\mathrm{mL}$ flask in a glovebox. Then, $10.0 \mathrm{~mL}$ of deionized water was added to the flask using a syringe. The reaction mixture was incubated at $80^{\circ} \mathrm{C}$ for $30 \mathrm{~min}$ to obtain a deep red clear solution. The freshly-made $\mathrm{NaHTe}$ solution was stored at room temperature prior to CdTe synthesis.

The NIR CdTe was synthesized according to a previously described method with minor modifications [26]. Briefly, $0.2 \mathrm{mmol}$ of $\mathrm{CdCl}_{2}$ was mixed with $0.34 \mathrm{mmol}$ of MPA in a $40 \mathrm{~mL}$ solution, with $\mathrm{pH}$ adjusted to 11.9 by dropwise addition of $2.0 \mathrm{M} \mathrm{NaOH}$. The reaction mixture was placed in a three-necked flask, bubbled with argon, and then heated to reflux $\left(100^{\circ} \mathrm{C}\right)$ under argon with a condenser attached. Under stirring conditions, freshly prepared $0.02 \mathrm{mmol}$ of $\mathrm{NaHTe}$ was added through a syringe to the $\mathrm{Cd}$ precursor solution at the boiling temperature. Samples were taken at different time intervals for measurement of UV-vis and photoluminescence spectra. 


\subsection{QDs characterizations}

The absorption and photoluminescence (PL) spectra of NIR CdTe were obtained using a Varian Cary 50 UV-visible spectrometer and a Hitachi F-4500 fluorescence spectrometer, respectively. Transmission electron microscopy (TEM) of NIR CdTe was performed on a Tecnai $\mathrm{G}^{2} \mathrm{~F} 20$ high-resolution transmission electron microscope at $200 \mathrm{kV}$. Dynamic light scattering (DLS) analyses of aqueous NIR CdTe was performed with a laser light scattering system (Nano ZS, Malvern). Photoluminescence quantum yields (QYs) were determined by measuring integrated fluorescence intensities of synthesized QDs and the reference using ICG (13\% in DMSO) as a standard [27]. Optical densities of synthesized QDs and standard dye solutions were measured using a Varian Cary-50 UV-vis spectrophotometer.

\subsection{NIR CdTe QDs functionalized with cRGD}

Based on carbodiimine chemistry, $\mathrm{EDC} \cdot \mathrm{HCl}$ was used as the cross-linker to prepare QD-peptide bioconjugates. The NIR CdTe QDs were reacted with cRGD (QDs/cRGD/EDC $\cdot \mathrm{HCl}$ molar ratio = 1:100:4000) in $0.01 \mathrm{M}$ phosphate buffer saline (PBS, pH 7.2) for $2 \mathrm{~h}$ at room temperature. The final bioconjugate products were purified by centrifugation at 100,000g for $30 \mathrm{~min}$ and washing with PBS for two times.

\subsection{Cancer targeting and imaging-guided sur- gery with NIR QD-bioconjugates}

The cancer targeting and imaging-guided surgery was performed on seven-week-old female athymic nude mice (Harlan) (25 g each). The animal procedures were in agreement with the guidelines of the Institutional Animal Care and Use Committee of Tongii University. The xenograft tumor model was established by subcutaneous injection of U87MG cells (ca. $5 \times 10^{6}$ per $50 \mu \mathrm{L}$ PBS) into the front flank of the mice. The mice were subjected to imaging studies when the tumor volume reached ca. $500 \mathrm{~mm}^{3}$ four weeks after inoculation. Before the tail vein injection, the mice were maintained with water only for $12 \mathrm{~h}$ to minimize autofluorescence from food.

Two hundred microliters of NIR QD-bioconjugates $(0.3 \mathrm{mg} / \mathrm{mL})$ were prepared in $2 \times$ PBS buffer and injected intravenously through the mouse tail. Video-rate NIR images were taken during and after the injection using a Fluobeam-700 NIR imaging system (Fluoptics, Grenoble, France). The power density of laser irradiation on tissues was ca. $1.5 \mathrm{~mW} / \mathrm{cm}^{2}$, the excitation wavelength was $690 \mathrm{~nm}$, and the exposure time was $100 \mathrm{~ms}$. For injection and subsequence imaging, the mice were anesthetized by intraperitoneal injection of $65 \mathrm{mg} / \mathrm{kg}$ pentobarbital. After the imaging-guided surgery, the fluorescence intensities of removed solid tumors were measured for comparison of signal intensity.

\section{Results and discussion}

\section{I. NIR QDs characterizations}

In the present study, fluorescent QDs with hydrodynamic diameters less than $10 \mathrm{~nm}$ were synthesized and used for IGS (Fig.1A), as those with bigger sizes could more easily be captured by reticuloendothelial system (RES) [28, 29]. After injected into bloodstream, the nanoparticles can be recognized as invaders by opsonin proteins, seized by macrophages, and eventually eradicated by the liver [30,31]. To avoid being recognized by the opsonin proteins, nanoparticles should be controlled less than $10 \mathrm{~nm}$ in hydrodynamic diameter [32, 33].

Presently, the application of nano-sized probes is considered as an innovative solution for early diagnosis and treatment of tumor. The percentage of injected nanoparticles that can arrive at tumor sites is mainly related to the capture by RES and the active-targeting capability of nanoparticles. As two competitive parts in a biological body, hepatic first-pass effect and active targeting can be manually adjusted by modifying the size, zeta potential and/or surface ligand of the nanoparticles [34]. The hydrodynamic diameter of CdTe QDs is around $4.5 \mathrm{~nm}$, below $10 \mathrm{~nm}$ (Fig.1B). And the DLS data shows no big aggregates in the aqueous CdTe QDs solution. The hydrodynamic diameter of bioconjugated QDs is nearly identical to that of naked QDs. The cRGD peptide is a kind of small molecule, which can not substantially change QDs' sizes and hydrodynamic diameters upon bioconjugation.

Optical fluorescence imaging techniques are non-invasive, real-time and convenient, thus have extensively been used in medical applications such as tumor detection, drug/gene delivery and drug discovery $[2,35]$. Practically, the contrast agents used for in vivo fluorescence imaging should be located in the NIR wavelength range and have high QYs and good photostability $[25,36]$. The sensitivity of in vivo tumor detection can be substantially improved by employing NIR fluorophores, which allow deep permeability, low absorption and autofluorescence by tissues. Furthermore, compared to visible fluorescent probes, NIR probes can be stimulated by longer wavelength sources, thus constraining the generation of singlet oxygen and reducing damage to biological components. Therefore, NIR dyes have increasingly been used for in vivo fluorescence imaging of tumors. Of 
note is that organic dyes have some inherent disadvantages that are not acceptable in intraoperative imaging, such as photobleaching after short-time observation. There are also difficulties in chemical modification of the molecular structure of the dyes, such as linking active chemical groups for post bioconjugation, and/or improving their water-solubility. To overcome these issues, NIR semiconductor QDs have been used and proven efficient for in vivo real-time fluorescence imaging [37-39].

NIR CdTe QDs were synthesized with maximum fluorescence emission peaks at $728 \mathrm{~nm}$ ( $38 \%$ of QY). These NIR QDs showed a sharp first excitonic absorption onset (Fig.1C). The ca. $30 \mathrm{~nm}$ stokes shift between the emission peak and associated first excitonic absorption onset indicated the dominance of band-edge luminescence from the QDs without deep trap emission at the long-wavelength side [26]. The good QY guaranteed the high sensitivity of in vivo tumor detection, as confirmed by their fine crystallinity in the high-resolution TEM images (Fig.1A in set).

The cytotoxicity test was conducted for NIR CdTe QDs on normal L929 cells. The 3-(4, 5-Dimethylthiazol-2-yl)-2, 5-diphenyltetrazolium bromide (MTT) results show low cytotoxicity of the CdTe QDs. The viabilities of L929 cells are over $70 \%$ when incubated with CdTe QDs at a concentration as high as $25 \mathrm{nmol} / \mathrm{L}$ for 24 hours (Fig. 1D). To reduce the toxicity of QDs, some methods are considered in our future research work, such as silica-coating, polymer-encapsulation and protein-capping.

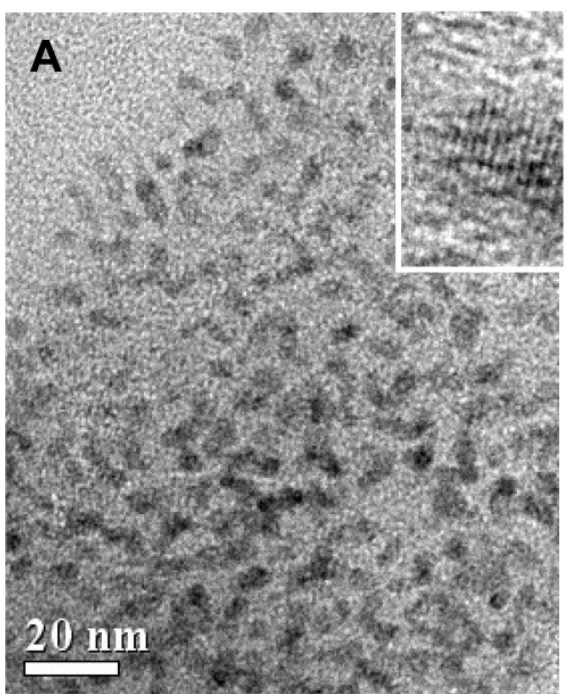

C

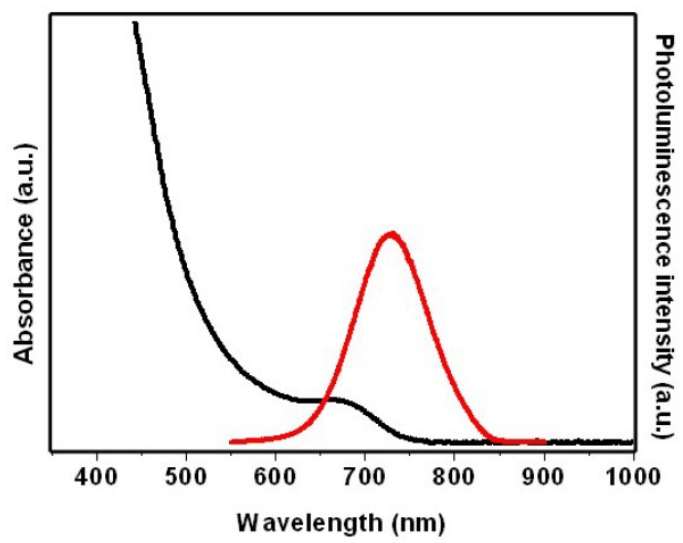

D
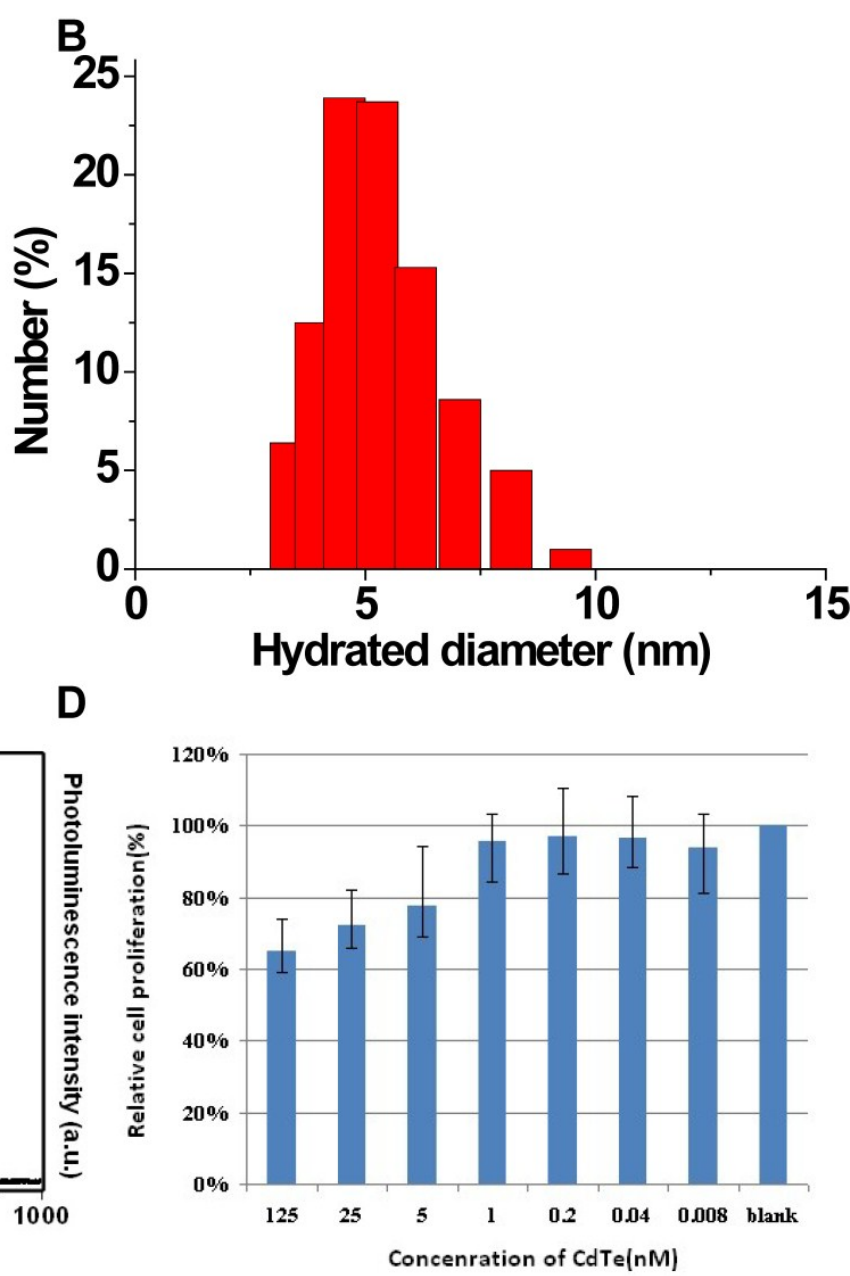

Fig.I TEM images (A), DLS (B), optical spectrum (C), and MTT assay (D) characterizations of the synthesized NIR CdTe QDs. 

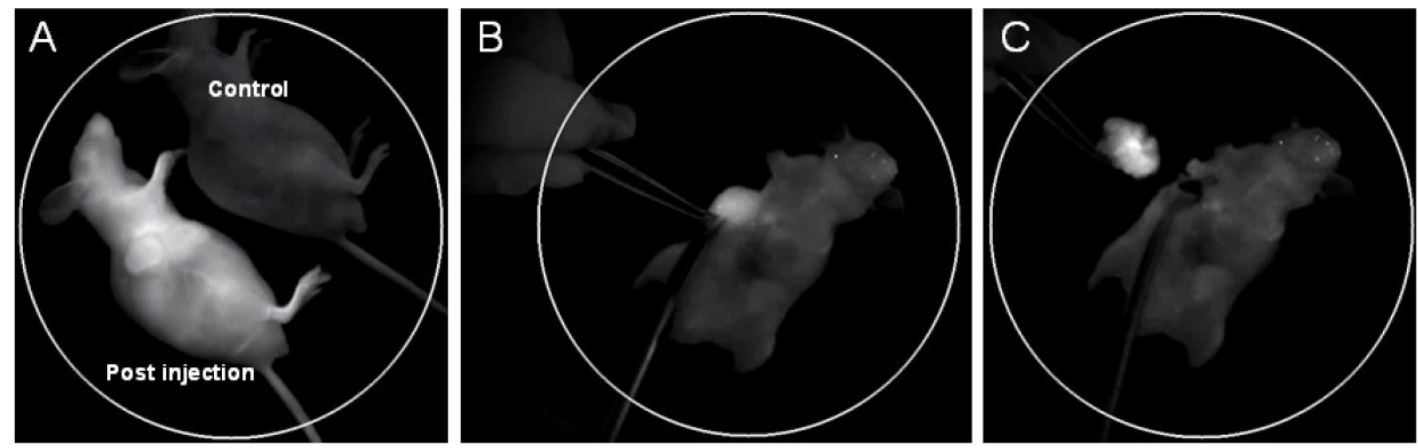

Fig. 2 (A) In vivo NIR fluorescence imaging of U87 MG tumor-bearing mice immediately after tail vein injection of cRGD-NIR QD bioconjugates (a non-injected mouse used as blank control); (B) NIR imaging-guided surgery performed $48 \mathrm{~h}$ after injection; and (C) the solid tumor precisely removed.

\subsection{Cancer targeting and imaging-guided surgery with NIR QD bioconjugates}

Targeting moieties (e.g. antibody, peptide and aptamer) are generally linked with nanoparticles for in vitro or in vivo medical specific detection. In the present study, cRGD was utilized for this purpose due to its higher affinity with U87 MG tumor, smaller moiety and higher availability compared with other highly specific antibodies. The cRGD-NIR QD bioconjugates were injected into U87 MG tumor-bearing mice via vein tails for both tumor-targeted imaging and IGS. The post-injection image of cRGD-NIR QD bioconjugates showed substantial NIR signal enhancement in the vessels (including tumor vessels) (Fig. 2A; also see Additional file 1: Supplementary Movie-S1). The signal was stronger at the tumor site than that of other body areas (Fig. 2A), and its intensity substantially increased during tumor-specific imaging. Subsequently, IGS was successfully performed to resect the detected tumor (Figs. 2B, 2C).

In clinical practice, cancer treatments include surgery, chemotherapy and radiation therapy, of which surgery is most frequently used for solid tumor resection. However, the efficiency of cancer surgery has been limited primarily due to the lack of precise guidance, the incomplete resection, the unanticipated wound, the difficulties in searching sentinel lymph node (SLN), and the prolonged time. These disadvantages can result in tumor recurrence and/or increase intraoperative risk. To improve the treatment regimens of cancer surgery, it is necessary to develop advanced imaging technologies [40], such as targeted probes and imaging systems for early precise diagnosis and tracking of tumor sites [41-43].

Without fluorescent dye labeling, the surgeons would have difficulty in marking the tumor margins, and either more or less marking could be harmful to the patients. It is largely due to this reason that the prognosis in advanced-stage cancer remains poor. Tumor-specific intraoperative NIR fluorescence imaging may improve the staging and debulking efforts in cytoreductive surgery, thereby improving the prognosis of related patients [6]. The application of IGS is particularly important and effective to patients with metastatic cancer, as cancer cells metastasizing via lymphatic system can also be intraoperatively eliminated during IGS. It has been shown that QDs are versatile for sentinel lymph node imaging [16, 44], and that the lymph nodes become clear when labeled with QDs [45]. Therefore, the cancer cells migrating in lymphatic basins can be easily removed.

\subsection{Active and passive cancer targeting demonstration}

To evaluate the targeting efficiency of cRGD-NIR CdTe QDs, non-targeted NIR CdTe QDs without cRGD were injected into U87 MG tumor-bearing mice for comparison purpose (Fig. 3). The signal intensity of active targeting via cRGD was nearly 5 times that of the passive targeting via enhanced permeability and retention (EPR) effect, although the latter slightly enhanced the signal intensity compared with the background signal of the non-injected blank control.

As mentioned above, the amount of injected nanoparticles can be elevated at the tumor site via active targeting, whereas the reduced size of nanoparticles can facilitate their escape from RES capture $[28,29]$. The utilization of targeting moiety-covered nanoparticles provides an active specificity for tumor detection, although the nanoparticles tend to accumulate at the tumor sites due to their inherent EPR effect [46-48].

In general, the newly formed tumor vessels were abnormal in form and architecture, with poorly-aligned defective endothelial cells and wide fenes- 
trations, whereas the tumor tissues were lack of effective lymphatic drainage. All these factors could lead to abnormal molecular and fluid transport dynamics, especially for nanoparticles and macromolecular drugs. The inherent features of solid tumor allow its vigorous growth, but can also be used for tumor diagnosis and treatment. Although passive targeting via EPR effect avoided the post surface modification of nanoparticles, we found that active targeting via tumor-specific moieties was more effective (Fig. 3). Additionally, the fluorescence emitting from the inside of the removed tumor tissue was found bright (Fig. 3B1), indicating that cRGD-NIR QD bioconjugates entered the tumor tissue through tumor vessels (Additional file 2: Supplementary Movie-S2). Our observations could be related to previous findings by Cai et al., who reported that QD705-RGD can retain inside the tumor [49].

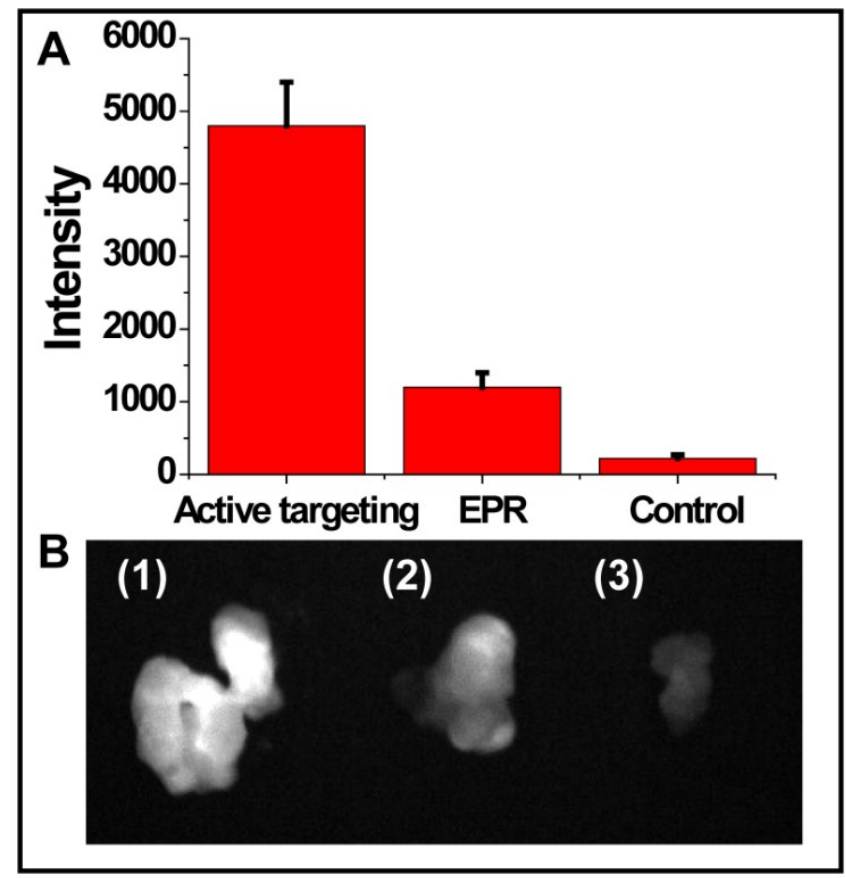

Fig. 3 (A) Quantification of fluorescence signals of the removed tumors detected by active targeting and EPR effect and the blank control (without injection), respectively; and (B) solid tumors excised from mice (I) active targeting, (2) EPR, and (3) blank control.

Angiogenesis, the formation of new blood vessels from preexisting vasculature, is essential for tumor growth and progression [50]. Integrin $\alpha_{v} \beta_{3}$ that binds RGD-containing components of the interstitial matrix such as vitronectin, fibronectin, and thrombospondin [51] plays a key role in tumor angiogenesis and metastasis[52, 53]. Being capable of noninvasively visualizing integrin $\alpha_{v} \beta_{3}$ expression, NIR QD-RGD probes has a great potential in cancer-targeted imaging of small preclinical tumor-bearing animal models and imaging-guided surgery. This is because the bright QD NIR signal can easily be detected at appropriate depth in small animals during cancer surgery. In this study, we found that specific nanoprobe contributed to the sensitivity of tumor detection. Thus, the surgeons were able to mark the tumor margins and further to remove them confidently with the help of nanoprobe (Additional file 3: Supplementary Movie-S3).

In addition to its medical application that provides real-time imaging guidance for surgeons during cancer surgery, NIR nano-sized probe also assists pathologists to study specific areas on various specimen, such as the sinus entry point of SLN and micrometastases in sectioned tissues [54, 55]. Although the safety of nanoparticles in vivo has not been well studied [30], nanoparticles and associated technology have displayed a brand-new perspective in cancer management. Considering the great anticancer potential of nanoparticles, it is imperative to explore new methods for improving the biocompatibility of nanoparticles. Once the safety of nanoparticles is addressed, the designed nanoparticles with multifunction such as imaging, selectivity, and safety can be applied for diagnosis and treatment of cancer.

\section{Conclusions}

At present, clinical experience with NIR fluorophores for intraoperative fluorescence imaging remains insufficient. Despite being the most frequently used NIR fluorophore, ICG is a non-targeted probe, which cannot be conjugated to tumor-specific ligands. By comparison, nanoparticles are inherently easier to be modified with ligands. In this study, we used the systemically administered tumor-targeted nano-sized NIR agent - cRGD-NIR QD bioconjugates for real-time fluorescence imaging. Our work highlighted the proof-of-principle and the potential benefit of intraoperative tumor-specific real-time fluorescence-targeted imaging and debulking surgery for integrin $\alpha_{v} \beta_{3}$-overexpressed tumor. Results showed that the active targeting of U87 MG tumor via cRGD-NIR QD bioconjugates was more effective than passive targeted imaging. Such synthesized cRGD-NIR QD bioconjugates function as universal NIR probes and play a role in tumor vasculature-targeted imaging of most types of cancer, because integrin $\alpha_{v} \beta_{3}$ is overexpressed in the majority of tumor vasculatures during angiogenesis. 


\section{Acknowledgements}

This work was supported by the National Natural Science Foundation of China (51003078, 81171393); the Nanotechnology Program of Shanghai Science \& Technology Committee (11nm0504500); the key project of basic research of Shanghai Science \& Technology Committee (10JC1412900); the Program for Young Excellent Talents in Tongji University (2009KJ072), and the Fundamental Research Funds for the Central Universities.

\section{Supplementary material}

Additional File 1:

Movie-S1: Whole body imaging of mice injected with NIR QDs. A non-injected mouse used as blank control for comparison purpose. The four bright spots on two mice caused by leakage of quantum dots in liquid droplets. http://www.thno.org/v02p0769s1.wmv Additional File 2:

Movie-S2: The solid tumors excised from mice. Note that quantum dot bioconjugates entered tumor tissues via active targeting.

http://www.thno.org/v02p0769s2.wmv

Additional File 3:

Movie-S3: NIR imaging-guided surgery.

http://www.thno.org/v02p0769s3.wmv

Additional File 4:

Cytotoxicity assay.

http://www.thno.org/v02p0769s4.pdf

\section{Competing Interests}

The authors have declared that no competing interest exists.

\section{References}

1. Tagaya N, Yamazaki R, Nakagawa A, Abe A, Hamada K, Kubota K, et al. Intraoperative identification of sentinel lymph nodes by near-infrared fluorescence imaging in patients with breast cancer. Am J Surg. 2008; 195: 850-3

2. Jiang S, Gnanasammandhan MK, Zhang Y. Optical imaging-guided cancer therapy with fluorescent nanoparticles. J R Soc Interface. 2010; 7: 3-18.

3. Renton SC, Gazet JC, Ford HT, Corbishley C, Sutcliffe R. The importance of the resection margin in conservative surgery for breast cancer. Eur J Surg Oncol. 1996; 22: 17-22.

4. Schiller DE, Le LW, Cho BCJ, Youngson BJ, McCready DR. Factors associated with negative margins of lumpectomy specimen: Potential use in selecting patients for intraoperative radiotherapy. Ann Surg Oncol. 2008; 15: 833-42.

5. Hadjipavlou AG, Kambin P, Lander PH, Crow WN, Simmons JW. Imaging guided minimally invasive surgery for low back pain sciatica and spinal infection. J Interv Radiol. 1999; 14: 1-22.

6. van Dam GM, Themelis G, Crane LMA, Harlaar NJ, Pleijhuis RG, Kelder $\mathrm{W}$, et al. Intraoperative tumor-specific fluorescence imaging in ovarian cancer by folate receptor-alpha targeting: first in-human results. Nat Med. 2011; 17: 1315-U202.

7. Zheng $\mathrm{XH}$, Xing $\mathrm{D}$, Zhou $\mathrm{FF}, \mathrm{Wu} \mathrm{BY}, \mathrm{Chen}$ WR. Indocyanine Green-Containing Nanostructure as Near Infrared Dual-Functional Targeting Probes for Optical Imaging and Photothermal Therapy. Mol Pharm. 2011; 8: 447-56.
8. Moghimi SM, Hunter AC, Murray JC. Nanomedicine: current status and future prospects. Faseb J. 2005; 19: 311-30.

9. Kumar B, Yadav PR, Goel HC, Rizvi MMA. Recent Developments in Cancer Therapy by the Use of Nanotechnology. Dig J Nanomater Bios. 2009; 4: 51-7.

10. Yezhelyev MV, Gao X, Xing Y, Al-Hajj A, Nie SM, O'Regan RM. Emerging use of nanoparticles in diagnosis and treatment of breast cancer. Lancet Oncol. 2006; 7: 657-67.

11. Kievit FM, Zhang MQ. Surface Engineering of Iron Oxide Nanoparticies for Targeted Cancer Therapy. Acc Chem Res. 2011; 44: 853-62.

12. Invernici G, Cristini S, Alessandri G, Navone SE, Canzi L, Tavian D, et al. Nanotechnology Advances in Brain Tumors: The State of the Art. Recent Pat Anticancer Drug Discov. 2011; 6: 58-69.

13. Groneberg DA, Giersig M, Welte T, Pison U. Nanoparticle-based diagnosis and therapy. Curr Drug Targets. 2006; 7: 643-8.

14. Gao JH, Chen K, Miao Z, Ren G, Chen XY, Gambhir SS, et al. Affibody-based nanoprobes for HER2-expressing cell and tumor imaging. Biomaterials. 2011; 32: 2141-8.

15. Yang $Q$, Gong $X$, Song $T$, Yang J, Zhu S, Li Y, et al. Quantum dot-based immunochromatography test strip for rapid, quantitative and sensitive detection of alpha fetoprotein. Biosens Bioelectron. 2011; 30: 145-50.

16. Tanaka E, Choi HS, Fujii H, Bawendi MG, Frangioni JV. Image-guided oncologic surgery using invisible light: Completed pre-clinical development for sentinel lymph node mapping. Ann Surg Oncol. 2006; 13: $1671-81$.

17. Liang GX, Gu MM, Zhang JR, Zhu JJ. Preparation and bioapplication of high-quality, water-soluble, biocompatible, and near-infrared-emitting CdSeTe alloyed quantum dots. Nanotechnology. 2009; 20: 415103.

18. Ruan G, Agrawal A, Marcus AI, Nie S. Imaging and tracking of tat peptide-conjugated quantum dots in living cells: new insights into nanoparticle uptake, intracellular transport, and vesicle shedding. J Am Chem Soc. 2007; 129: 14759-66.

19. Peng XG, Manna L, Yang WD, Wickham J, Scher E, Kadavanich A, et al. Shape control of CdSe nanocrystals. Nature. 2000; 404: 59-61.

20. Kim GB, Kim YP. Analysis of Protease Activity Using Quantum Dots and Resonance Energy Transfer. Theranostics. 2012; 2: 127-38.

21. Yezhelyev MV, Qi LF, O'Regan RM, Nie S, Gao XH. Proton-sponge coated quantum dots for siRNA delivery and intracellular imaging. J Am Chem Soc. 2008; 130: 9006-12.

22. Xing Y, Chaudry Q, Shen C, Kong KY, Zhau HE, WChung L, et al. Bioconjugated quantum dots for multiplexed and quantitative immunohistochemistry. Nat Protoc. 2007; 2: 1152-65.

23. Medintz IL, Uyeda HT, Goldman ER, Mattoussi H. Quantum dot bioconjugates for imaging, labelling and sensing. Nat Mater. 2005; 4: 435-46.

24. Jaiswal JK, Mattoussi H, Mauro JM, Simon SM. Long-term multiple color imaging of live cells using quantum dot bioconjugates. Nat Biotechnol. 2003; 21: 47-51.

25. Lim YT, Kim S, Nakayama A, Stott NE, Bawendi MG, Frangioni JV. Selection of quantum dot wavelengths for biomedical assays and imaging. Mol Imaging. 2003; 2: 50-64.

26. Zou L, Gu ZY, Zhang N, Zhang YL, Fang Z, Zhu WH, et al. Ultrafast synthesis of highly luminescent green- to near infrared-emitting CdTe nanocrystals in aqueous phase. J Mater Chem. 2008; 18: 2807-15.

27. Hu DH, Zhang PF, Gong P, Lian SH, Lu YY, Gao DY, et al. A fast synthesis of near-infrared emitting $\mathrm{CdTe} / \mathrm{CdSe}$ quantum dots with small hydrodynamic diameter for in vivo imaging probes. Nanoscale. 2011; 3 : 4724-32.

28. De Jong WH, Hagens WI, Krystek P, Burger MC, Sips AJ, Geertsma RE. Particle size-dependent organ distribution of gold nanoparticles after intravenous administration. Biomaterials. 2008; 29: 1912-9.

29. Schipper ML, Iyer G, Koh AL, Cheng Z, Ebenstein Y, Aharoni A, et al. Particle Size, Surface Coating, and PEGylation Influence the Biodistribution of Quantum Dots in Living Mice. Small. 2009; 5: 126-34.

30. Sharifi S, Behzadi S, Laurent S, Laird Forrest M, Stroeve P, Mahmoudi M. Toxicity of nanomaterials. Chem Soc Rev. 2012; 41: 2323-43.

31. Lynch I, Dawson KA. Protein-nanoparticle interactions. Nano Today. 2008; 3: 40-7.

32. Gao JH, Chen $\mathrm{K}$, Xie RG, Xie J, Lee S, Cheng Z, et al. Ultrasmall Near-Infrared Non-cadmium Quantum Dots for in vivo Tumor Imaging. Small. 2010; 6: 256-61.

33. Liu Q, Sun $Y$, Yang TS, Feng W, Li CG, Li FY. Sub-10 nm Hexagonal Lanthanide-Doped NaLuF(4) Upconversion Nanocrystals for Sensitive Bioimaging in Vivo. J Am Chem Soc. 2011; 133: 17122-5.

34. Longmire MR, Ogawa M, Choyke PL, Kobayashi H. Biologically optimized nanosized molecules and particles: more than just size. Bioconjug Chem. 2011; 22: 993-1000. 
35. Hawrysz DJ, Sevick-Muraca EM. Developments toward diagnostic breast cancer imaging using near-infrared optical measurements and fluorescent contrast agents. Neoplasia. 2000; 2: 388-417.

36. Gioux S, Choi HS, Frangioni JV. Image-Guided Surgery Using Invisible Near-Infrared Light: Fundamentals of Clinical Translation. Mol Imaging. 2010; 9: 237-55.

37. Park J, Dvoracek C, Lee KH, Galloway JF, Bhang HEC, Pomper MG, et al. CuInSe/ZnS Core/Shell NIR Quantum Dots for Biomedical Imaging. Small. 2011; 7: 3148-52.

38. Kim JS, Cho KJ, Tran TH, Nurunnabi M, Moon TH, Hong SM, et al. In vivo NIR imaging with CdTe/CdSe quantum dots entrapped in PLGA nanospheres. J Colloid Interf Sci. 2011; 353: 363-71.

39. Gao J, Chen $X$, Cheng Z. Near-infrared quantum dots as optical probes for tumor imaging. Curr Top Med Chem. 2010; 10: 1147-57.

40. Peters TM. Image-guided surgery: from $X$-rays to virtual reality. Comput Methods Biomech Biomed Engin. 2000; 4: 27-57.

41. Hattori A, Suzuki N, Otori N, Iimura J, Moriyama H. Development of a real-time image-guided surgery system for stereo-endoscopic sinus surgery. Stud Health Technol Inform. 2009; 142: 112-6.

42. Moon WK, Lin Y, O'Loughlin T, Tang Y, Kim DE, Weissleder R, et al. Enhanced tumor detection using a folate receptor-targeted near-infrared fluorochrome conjugate. Bioconjug Chem. 2003; 14: 539-45.

43. Chen X, Conti PS, Moats RA. In vivo near-infrared fluorescence imaging of integrin alphavbeta3 in brain tumor xenografts. Cancer Res. 2004; 64: 8009-14.

44. Pons T, Pic E, Lequeux N, Cassette E, Bezdetnaya L, Guillemin F, et al. Cadmium-Free CuInS(2)/ZnS Quantum Dots for Sentinel Lymph Node Imaging with Reduced Toxicity. Acs Nano. 2010; 4: 2531-8.

45. Kobayashi H, Hama Y, Koyama Y, Barrett T, Regino CAS, Urano Y, et al. Simultaneous multicolor imaging of five different lymphatic basins using quantum dots. Nano Lett. 2007; 7: 1711-6.

46. Tamilvanan S. Formulation of multifunctional oil-in-water nanosized emulsions for active and passive targeting of drugs to otherwise inaccessible internal organs of the human body. Int J Pharmaceut. 2009; 381: 62-76.

47. Danhier F, Feron O, Preat V. To exploit the tumor microenvironment: Passive and active tumor targeting of nanocarriers for anti-cancer drug delivery. J Control Release. 2010; 148: 135-46.

48. Huynh NT, Roger E, Lautram N, Benoit JP, Passirani C. The rise and rise of stealth nanocarriers for cancer therapy: passive versus active targeting. Nanomedicine-Uk. 2010; 5: 1415-33.

49. Cai WB, Shin DW, Chen K, Gheysens O, Cao QZ, Wang SX, et al. Peptide-labeled near-infrared quantum dots for imaging tumor vasculature in living subjects. Nano Lett. 2006; 6: 669-76.

50. Wong MLH, Prawira A, Kaye AH, Hovens CM. Tumour angiogenesis: Its mechanism and therapeutic implications in malignant gliomas. J Clin Neurosci. 2009; 16: 1119-30.

51. Xiong JP, Stehle T, Zhang R, Joachimiak A, Frech M, Goodman SL, et al. Crystal structure of the extracellular segment of integrin alpha Vbeta3 in complex with an Arg-Gly-Asp ligand. Science. 2002; 296: 151-5.

52. Ye YP, Chen XY. Integrin Targeting for Tumor Optical Imaging. Theranostics. 2011; 1: 102-26.

53. Millard M, Odde S, Neamati N. Integrin Targeted Therapeutics. Theranostics. 2011; 1: 154-88.

54. Yezhelyev MV, Al-Hajj A, Morris C, Marcus AI, Liu T, Lewis M, et al. In situ molecular profiling of breast cancer biomarkers with multicolor quantum dots. Adv Mater. 2007; 19: 3146-51.

55. Peng CW, Li Y. Application of Quantum Dots-Based Biotechnology in Cancer Diagnosis: Current Status and Future Perspectives. J Nanomater. 2010; :676839. 